\title{
N400-like Potentials and Reaction Times Index Semantic Relations between Highly Repeated Individual Words
}

\author{
Louis Renoult and J. Bruno Debruille
}

\begin{abstract}
The N400 ERP is an electrophysiological index of semantic processing. Its amplitude varies with the semantic category of words, their concreteness, or whether their meaning matches that of a preceding context. The results of a number of studies suggest that these effects could be markedly reduced or suppressed for stimuli that are repeated. Nevertheless, we have recently shown that significant effects of semantic matching and category could be obtained on N400-like potentials elicited by massively repeated target words in a prime-target semantic categorization task. If such effects could be obtained when primes also are repeated, it would then be possible to study the semantic associations between individual words. The present study thus aimed to test this hypothesis while (1) controlling for a potential contribution of physical matching to the processing of repeated targets and (2) testing if the N400-like effects obtained in these conditions are modulated by task instruction, as are classic N400 effects. Two category words were used as primes and two
\end{abstract}

exemplars as targets. In one block of trials, subjects had to respond according to the semantic relation between prime and target (semantic instruction) and, in another block, they had to report changes in letter case (physical instruction). Results showed that the amplitude of the N400-like ERP obtained was modulated by semantic matching and category but not by letter case. The effect of semantic matching was observed only in the semantic instruction block. Interestingly, the effect of category was not modulated by task instruction. An independent component analysis showed that the component that made the greatest contribution to the effect of semantic matching in the time window of the N400-like potential had a scalp distribution similar to that reported for the $\mathrm{N} 400$ and was best fit as a bilateral generator in the superior temporal gyrus. The use of repetition could thus allow, at least in explicit semantic tasks, a drastic simplification of N400 protocols. Highly repeated individual words could be used to study semantic relations between individual concepts.

\section{INTRODUCTION}

Semantic memory is defined as the knowledge we have of the world, including facts and concepts (Tulving, 1972). The semantic representation of an object is what characterizes this object as a distinct entity. The activation of this representation depends on the meaning of the item and not on the physical format of its presentation (Martin, 2001). Our knowledge of the organization of semantic representations in the brain has greatly benefited from the use of neuroimaging techniques. Notably, an ERP, the N400, has proved to be of major interest for the study of semantic memory. The N400 corresponds to a negative deflection which develops between 250 and 500 msec after the onset of a stimulus and constitutes a response of the brain to any potentially meaningful item (for a review, see Kutas, Van Petten, \& Kluender, 2006). The amplitude of the N400 is modulated by a number of semantic manipulations. It is decreased for primed or congruous words compared with unprimed or incongruous words in sentence (Kutas \& Hillyard, 1980, 1984) or prime-target (Bentin, McCarthy, \& Wood, 1985) contexts. These effects of priming and semantic congruity are observed even when access to the

McGill University, Montréal, Québec, Canada

meaning of words is not necessary, such as in lexical decision tasks (e.g., Bentin et al., 1985). However, they are of larger amplitude when subjects have to focus on the semantic properties of words (Chwilla, Brown, \& Hagoort, 1995; Mitchell et al., 1991; Holcomb, 1988). Another replicated modulation of ERPs in the N400 time window is the effect of concreteness. N400s to concrete words have larger amplitudes than those evoked by abstract words (West \& Holcomb, 2000; Holcomb, Kounios, Anderson, \& West, 1999; Kounios \& Holcomb, 1994). Moreover, within concrete words, different distributions of the N400 amplitude on the scalp have been associated with words belonging to living and artifactual categories (Kiefer, 2001, 2005; Sim \& Kiefer, 2005).

N400 amplitude is also sensitive to stimulus repetition. It is smaller for words that have been presented previously compared with words that occur for the first time in an experiment (for a review, see Rugg, 1995). This repetition effect is larger than the semantic priming effect (Holcomb, Reder, Misra, \& Grainger, 2005; Rugg, 1985). The results of some studies suggest that this reduction of N400 amplitude is proportional to the number of repetitions (Finnigan, Humphreys, Dennis, \& Geffen, 2002; Rugg, Brovedani, \& Doyle, 1992; Van Petten, Kutas, Kluender, Mitchiner, \& McIsaac, 1991). In contrast, other studies 
reported that $\mathrm{N} 400$ amplitude did not decrease further after two or three presentations (Van Strien, Hagenbeek, Stam, Rombouts, \& Barkhof, 2005; Kazmerski \& Friedman, 1997; Besson, Kutas, \& Van Petten, 1992; Kounios \& Holcomb, 1992; Young \& Rugg, 1992).

N400 amplitude is, therefore, modulated both by semantic manipulations and repetition. Moreover, a number of studies have shown that the effect of repetition interacts with that of semantic congruity (Mitchell, Andrews, \& Ward, 1993; Besson et al., 1992), semantic category (Kiefer, 2005; Sim \& Kiefer, 2005), and concreteness (Kounios \& Holcomb, 1994). More specifically, these effects were shown to be reduced or suppressed when stimuli were repeated. For the effect of concreteness, the interaction with repetition was found to be task-dependent. Kounios and Holcomb (1994) observed that the N400 difference between concrete and abstract words was eliminated by repetition in a lexical decision task but simply reduced in a concreteabstract classification task. As to category effects, they were found to be smaller (Sim \& Kiefer, 2005) or to vanish (Kiefer, 2005) for stimuli that had already been presented. The task used could also influence the interaction of category effects with repetition. Category effects were shown to be more reliable in explicit semantic tasks (Devlin et al., 2002). It is thus possible that the marked reduction of these effects with repetition was due to the use of a shape judgment task in Sim and Kiefer (2005) and of a lexical decision task in Kiefer (2005). As to the effect of semantic congruity on the N400, two studies using words in sentences found an interaction with repetition (Mitchell et al., 1993; Besson et al., 1992). In Besson et al. (1992), where two levels of repetitions were contrasted, the difference between semantically congruous and incongruous sentence endings was attenuated at the second presentation and completely vanished at the third presentation of the sentences. However, in this study, complete sentences were repeated, making it likely that their final words, to which N400s were recorded, could be anticipated. These words would thus have been completely primed. In accordance with this interpretation, a number of studies have found that when words in sentences are repeated in a context different from that of their initial presentation, the reductions in raw N400 amplitude and in the effect of priming are smaller or absent (Besson \& Kutas, 1993; Mitchell et al., 1993; Woodward, Ford, \& Hammett, 1993).

The rationale for the present study is based on the idea that semantic congruity effects could also resist repetition in experiments using prime-target word pairs provided that a logic similar to that used in these latter sentence paradigms is followed. Target words can be repeated independently of prime words and the task can explicitly require access to semantic representations, as when processing sentences for meaning. We have recently made a first attempt at testing this hypothesis (Debruille \& Renoult, 2009). In this study, two versions of a semantic categorization task were contrasted. The first was a "classic" design in which two category words were used as primes and numerous nonrepeated exemplars of these categories as targets. In the second version of the task, primes and targets were inverted: The primes were the numerous nonrepeated category exemplars and the target words, to which N400s were recorded, were simply the two category words, each presented 60 times. Importantly, as categories and exemplars can be associated with different N400 raw amplitudes (Kounios \& Holcomb, 1992), only the persistence of N400 effects with repetition was analyzed. Results showed that the effects of semantic matching ${ }^{1}$ and of semantic category on RT and N400 obtained in the two versions of the task were similar. The distribution on the scalp of the ERP effects in the condition where targets were massively repeated condition resembled that observed in the condition in which they were not repeated. Also, similar clusters of independent components (ICs) accounted for the match effect of the two task versions.

The results of this experiment thus provided a strong support to the idea that the semantic processes indexed by the N400 could occur and be measured for highly repeated words. However, the use of various nonrepeated primes in this study likely resulted in processing requirements that were not very different from those involved in more "classic" semantic categorization tasks. If similar results could be obtained when primes also are repeated, then it would constitute a more definitive evidence of the resistance of semantic processing to repetition. Moreover, this could have important consequences as it would suggest that the semantic processes indexed by the $\mathrm{N} 400$ could be studied for individual words and their associations. It may be argued that an ERP other than the N400 has already been proposed for the study of individual repeated words (reviewed in Pulvermüller \& Shtyrov, 2006). However, this potential, the MMN, reflects different processes, notably acoustic change detection, and is evoked in different types of paradigms. Thus, it may be of critical importance to also test whether N400 processes can be triggered by the presentation of individual words. This would allow a drastic simplification of semantic paradigms. In addition, subjective variations in associations could be directly investigated. This could be of interest not only to refine classic norms of associations based on questionnaires but also to investigate implicit associations such as those involved by personal judgments, attitudes (Lust \& Bartholow, 2009), or social stereotypes (Blair \& Banaji, 1996). Moreover, it would provide a solution for heterogeneity problems. Experimental protocols can be restricted to the most typical exemplars of semantic categories. Nevertheless, to show that this could actually be possible, one has first to demonstrate that N400 effects can be obtained when using not only highly repeated targets but also highly repeated primes.

In addition, it should also be verified that the N400-like potential evoked in these repeated conditions does not depend on nonsemantic processing, such as a physical matching. Given that only two target words were used in the repeated target version of the task of Debruille and Renoult (2009), subjects may have simply retrieved 
the decision and the associated response at each presentation. This retrieval could have occurred as soon as a match or a mismatch was detected between the physical features of the actual target word and the physical features of the expected target word. This may have prevented a deep processing of target words. Although the aforementioned similarity of the effects obtained for repeated and nonrepeated targets makes this possibility unlikely, a partial contribution of physical matching cannot be excluded. This interpretation is supported by the early peak latency (290 $\mathrm{msec}$ ) of the N400-like potential obtained for the highly repeated targets. This early latency is reminiscent of that of components of the N200 family, the amplitude of which increases with visual deviations from a template (for a recent review, see Folstein \& Van Petten, 2008). These negativities peak between 200 and $350 \mathrm{msec}$ after stimulus onset and have a fronto-central distribution. They are evoked by simple (e.g., single letters) or more complex stimuli (faces, geometric shapes) that physically mismatch a reference stimulus or deviate from a category of stimuli. N200s are commonly observed not only in oddball tasks but also in sequential matching tasks, where match and mismatching trials have equal probabilities of occurrence. However, it is to note that the onset latency of an ERP deflection is often considered as a better reflection of the timing of a cerebral process than its peak latency (Kutas et al., 2006; Picton et al., 2000). Accordingly, the fact that the onset of the early N400-like potential found in Debruille and Renoult (2009) was the same as that of the N400 observed in the nonrepeated condition (i.e., around $230 \mathrm{msec}$ postonset) goes against the interpretation of this negativity as an N200 and supports an N400 interpretation. Nevertheless, N400s peaking as early as $290 \mathrm{msec}$ have not often been reported. One exception can be found in a study by Simon, Bernard, Largy, Lalonde, and Rebai (2004). These authors described an N280 in a lexical decision task using three massively repeated words and asked whether this ERP could be an N400. Similarly, using an auditory sentence paradigm with six possible final words, Karniski, Vanderploeg, and Lease (1993) obtained a small effect of semantic congruity on a centro-parietal negativity peaking about $280 \mathrm{msec}$ after word onset. The authors interpreted this effect as a modulation of $\mathrm{N} 400$ but discussed the possibility that it might be an N200. The use of auditory presentation in this study may however have allowed participants to categorize final words with just their first phonemes and could also explain this early latency. In any case, to ensure that the early negative ERP observed in our previous study is related to the N400 and does not include an N200 component, it appears necessary to compare semantic matching with physical matching.

Interestingly, this comparison provides the opportunity to verify if the amplitude of the potential seen as an N400 in conditions of massive repetition is modulated by task instruction as are "classic" N400 effects. These effects are minimal or absent when the task pertains to the physical features of words, intermediate for lexical deci- sion tasks and maximum for semantic tasks (Chwilla et al., 1995; Deacon, Breton, Ritter, \& Vaughan, 1991; Rugg, 1984). Therefore, if the effects interpreted as semantic matching effects are N400 effects, they should be much smaller or absent in a physical task. Alternatively, if these effects include N200 effects, as mentioned above, they should be greater in physical tasks, as in Deacon et al. (1991), or at least of similar magnitude, as in Kramer and Donchin (1987). The physical task chosen for the present study was a letter case discrimination (Chwilla et al., 1995; Deacon et al., 1991; Rugg, 1984). This task is commonly used to contrast brain processes that are sensitive to the physical aspect of words with those sensitive to their abstract identity (e.g., Dehaene et al., 2001, 2004).

In sum, the main hypothesis of the present study was that the N400-like effects obtained with highly repeated target words in a prime-target semantic categorization task would be observed when primes also are highly repeated and thus with only a few pairs of words used throughout the experiment. The two other hypotheses were that N400-like effects would not be modulated by physical matching but that they would vary with task instruction as genuine N400 effects. If these three hypotheses were to be confirmed, it would provide a strong support for the idea that the semantic processes indexed by $\mathrm{N} 400$ resist high repetition rates and may be explored to study the associations between individual words. To test these hypotheses, we used a design with only four different words: Primes were one of two category words, and targets were one of two exemplar words. There were thus four possible prime-target pairs. Letter case was manipulated so that the physical aspects of targets either matched that of the primes or did not. The effect of task instruction was examined by asking subjects to compare targets with primes as to their meaning or as to their physical aspect. An independent component analysis (ICA) was used to compare the components accounting for the effect of letter case matching with those accounting for the effect of semantic matching.

\section{METHODS \\ Participants}

Twenty-three right-handed native French speakers (17 women) took part in this experiment. They had normal or corrected-to-normal vision and no history of neurological or psychiatric disorders. They were recruited by newspaper advertisements among people aged between 18 and 30 years (mean $=24$ years, $S D=4$ years) who had at least a college level of education. They signed an informed consent form accepted by the Douglas Institute Research and Ethics Board.

\section{Task and Procedure}

Subjects were seated comfortably in a dimly lit room in front of a computer screen placed $1 \mathrm{~m}$ from their eyes. 
Black words, written in Boston 15 font, were presented at the center of the screen on a white background. Each trial was made of three serially presented French words, each occurring for a 1-sec duration and followed by a 1-sec blank interval. The word "attention" (which has the same spelling and meaning in French and English) initiated each trial, followed by the prime word and the target word. The primes were the category words "animal" (same spelling and meaning as in English) and "chose" (i.e., "thing" in English), presented in lowercase. The target words were "table" (same spelling and same meaning as in English) and "chien" (i.e., "dog"). In half of the trials, the target word semantically matched the prime word. In the other half, it did not. Target words were presented in lowercase or uppercase at random with equal probability (0.5). Then, 1 to $1.5 \mathrm{sec}$ after the offset of the target stimulus, a blink instruction, "clignez des yeux," appeared. The next trial began after a time interval that randomly varied between 1.5 and 2 sec.

The two target words, "table" and "chien," were chosen as they had the same number of letters, were very typical exemplars of the artifact and animal categories, and had similar psycholinguistic characteristics. The mean base-10 logarithm of the frequency of usage of "table" is 4.4 and that of "chien" is 4.08, according to the Brulex database (Content, Mousty, \& Radeau, 1990). In the 5-point rating scales used in the norms of Bonin et al. (2003), where 5 represents the maximum, their concreteness ratings are 4.9 and 4.8, their imageability ratings are 4.7 and 4.9, and their subjective frequency ratings are 4.6 and 4.5 , respectively, the latter measure being highly correlated with familiarity (e.g., $r=.9$ in Robin, 2006).

There were two blocks of trials, one with a semantic instruction and one with a physical instruction. The letter case of target words was varied in both blocks. In the semantic instruction block, subjects had to respond according to whether the target word semantically matched the category of the prime word. In the physical instruction block, they had to respond according to whether the target word had the same letter case as the prime. In both blocks, they had to answer as rapidly and as accurately as possible by pressing one of two keyboard keys with their right index finger. There were eight trial types in each block ( 2 primes $\times 2$ targets $\times 2$ letter cases). Each trial type included 50 trials. Each word, prime or target, was thus presented 200 times in each block. Hereafter, we used the term "artifact targets" to refer to the trials where the word "table" was presented and the term "animal targets" to refer to the trials where the word "chien" (i.e., "dog") was presented.

Fifteen of the 23 subjects went through both blocks of trials, half of them $(n=7)$ starting with the semantic instruction and the other half $(n=8)$ with the physical instruction. Eight other subjects only had the block with the semantic instruction. This allowed us to have a total of 15 subjects who completed both block of trials and also 15 subjects who had the semantic block first (and therefore who had not viewed the words in the other block before). The data of this last group of subjects were used to verify that the semantic matching effect on N400 and RT did not vary as a function of the number of repetitions.

\section{Data Acquisition}

Accuracy and RT were recorded for each trial. The EEG was recorded with tin electrodes mounted in an elastic cap (Electrocap International) from 30 active points, all referenced to the right ear lobe. Twenty-eight of these points were placed according to the extended International 10-20 System (Electrode Nomenclature Committee, 1991). These electrode sites could be grouped in a sagittal subset, which comprised Fz, FCz, Cz, and Pz; a parasagittal subset, including FP1/2, F3/4, FC3/4, C3/4, $\mathrm{CP} 3 / 4, \mathrm{P} 3 / 4$, and $\mathrm{O} 1 / 2$; and a lateral subset, including F7/8, FT7/8, T3/4, TP7/8, and T5/6. The remaining two active electrodes were placed below each eye to allow the monitoring of vertical eye movements by comparing their EEG signals with those derived from FP1 and FP2. The monitoring of horizontal eye movements was done by comparing F8 with F7 signals. The impedance was kept below $5 \mathrm{k} \Omega$. The EEG was amplified 20,000 times by Contact Precision amplifiers. High- and low-pass filter half-amplitude cutoffs were set at .01 and $100 \mathrm{~Hz}$, with an additional $60-\mathrm{Hz}$ electronic notch filter. Signals were then digitized on-line at a sampling rate of $256 \mathrm{~Hz}$ and stored along with stimulus and response codes for subsequent averaging using the Instep (version 4.3) software package.

EEG epochs contaminated by eye movements, excessive myogram, amplifier saturations, or analog to digital clippings were removed off-line by setting automatic rejection criteria. Trials for which analog to digital clipping exceeded a 100-msec duration, and electrodes for which amplitude exceeded $\pm 100 \mu \mathrm{V}$ were excluded from averaging. We then further ensured that the signals recorded by frontal electrodes were not contaminated by ocular activity. For vertical eye movements, this was done by comparing the activity recorded by FP1/2 channels with that recorded by the electrodes placed below each eye, subject by subject and condition by condition, looking for polarity inversions. Similarly, for horizontal eye movements, we compared F8 signal with F7 signal and looked for polarity inversions that could signal ocular activity. No subject had to be reaveraged after this inspection.

\section{Data Processing and Measures}

Mean RTs for each condition were computed using only the correct responses and excluding the trials where subjects took more than $2000 \mathrm{msec}$ to respond. ERPs to target words were computed by averaging the 1000 msec EEG epochs of these trials in each experimental condition, using a -200 - to 0-msec baseline before target onset.

In addition to that of the N400s, the amplitudes of the P200s and that of the late positive complexes (LPCs) were measured because variations of the ERPs in these latter 
time windows can be seen in physical matching tasks. For measuring these three potentials, we computed the mean voltage respective to the baseline in time windows that were chosen with a midpeak latency technique, also referred to as the fractional latency approach in the guidelines of Picton et al. (2000). This method has the advantage of focusing on particular deflections, whereas focusing on specific effects may bias statistical analyses. For the N400, we first measured the latency of the negative peak that appeared between 200 and $500 \mathrm{msec}$ at $\mathrm{Cz}$ electrode on the grand average of semantic mismatch trials. This negative peak culminated around $330 \mathrm{msec}$ after target presentation, similar to that found by Debruille and Renoult (2009). As in this study, we will refer to this negativity as an N400-like potential. We then measured the peak latency of the preceding P200. This latency was added to the latency of the N400-like potential, and this sum was divided by 2 . The result was used as the onset of the N400-like potential time window. Similarly, the peak latency of the N400-like potential was added to that of the LPC, and the result was divided by 2 to obtain the offset of the N400-like potential time window. The window of measure of the P200 and LPC were determined with the same technique, except that peak latencies were measured on the grand average of physical mismatch trials at $\mathrm{Cz}$ for the P200 and at Pz for the LPC where their amplitudes were respectively maximal. Time windows of measures of the P200 and LPC had then to be slightly recentered (by about $10 \mathrm{msec}$ ) to prevent an overlap with the N400-like potential time window. For the P200, the time window was 155 to $265 \mathrm{msec}$, for the N400-like potential 265 to $405 \mathrm{msec}$, and for the LPC 405 to $550 \mathrm{msec}$.

\section{Statistical Analyses}

For analyzing RTs, we ran a repeated measures ANOVA with a multivariate approach. It had four within-subject factors: Target Category (animal vs. artifact), Match (semantic match vs. semantic mismatch), Letter Case (same case as prime vs. different case), and Instruction (semantic vs. physical).

For each of the three time windows of ERP measures, three repeated measures ANOVAs with multivariate approaches were performed with the same within-subjects factors as the RT analysis plus the electrode factor. One of these three ANOVAs was for the sagittal subset of electrodes. The two others were for the parasagittal and the lateral subsets, for which another within-subject factor, Hemiscalp (right vs. left), was added. The Greenhouse and Geisser (1959) procedure was used to compensate for possible violations of the sphericity assumption associated with the electrode factor that had more than two levels. In this case, the original degrees of freedom are reported together with the epsilon $(\varepsilon)$ and the corrected probability level.

\section{Independent Component Analysis (ICA)}

In an attempt to compare the ERP generators of the effect of semantic matching with that of the effect of physical matching (i.e., letter case), ERPs of individual subjects for each condition were submitted to an ICA. ICA decomposes the unaveraged EEG (Jung et al., 2001) or ERPs (Dien, Khoe, \& Mangun, 2007; Makeig et al., 1999) into a sum of ICs with fixed scalp maps, and maximally independent time course (Makeig, Jung, Bell, Ghahremani, \& Sejnowski, 1997). For biologically plausible components, these component maps have been shown to nearly fit the projection of a single equivalent current dipole (ECD), allowing hypotheses as to the localization of this dipole (Onton, Westerfield, Townsend, \& Makeig, 2006).

The ICA was conducted with EEGLAB 6.01b (Delorme \& Makeig, 2004), a freely available open source toolbox (http://www.sccn.ucsd.edu/eeglab), running under Matlab 7.7 (MathWorks, Natick, MA). Individual subjects ERPs of 1 second ( -200 to 800 msec after target onset) from our 30 active electrodes were concatenated separately for each condition (semantic match, semantic mismatch, letter case match, and letter case mismatch), yielding four different matrices of $30 \times 3855$ points $(15$ subjects $\times 257$ time points) that were submitted to the same ICA. Specifically, ERPs for the two semantic conditions were taken from the semantic instruction block averaged across the two target categories, and ERPs for the two physical conditions were taken from the physical instruction block also averaged across the two target categories. We applied infomax ICA (Bell \& Sejnowski, 1995) with the EEGLAB function runica (Delorme \& Makeig, 2004). The PCA option of runica was used as a preprocessing step to reduce data dimensionality and prevent individual differences to influence the decomposition (Dien et al., 2007). Fifteen factors were retained, as a prior PCA revealed that seven and eight factors accounted for $95 \%$ of variance of the semantic effect and the physical effect, respectively.

We then used the envtopo() function of EEGLAB (Onton et al., 2006; Delorme \& Makeig, 2004) to identify the five ICs that made the largest contribution to the effects of semantic matching (i.e., semantic mismatch - semantic match) and letter case (different letter case - same letter case as the prime) in each of the time window of measures of ERPs, that is, that of the P200 (155-265 msec after target onset), N400-like potential (265-405 msec), and LPC (405$550 \mathrm{msec}$ ). This allowed us to identify 10 different ICs whose activity was modulated by one and/or the other effect in one or several of the windows of measures. The activity of each of these ICs in each time window was then submitted to a repeated measures ANOVA with Match (semantic match vs. semantic mismatch) and Letter Case (same case as prime vs. different case) as within-subject factors. Finally, source localization was computed for all of these 10 ICs with DIPFIT2, an EEGLAB plug-in that performs component localization by fitting an ECD model using a nonlinear optimization technique (Scherg, 1990). ICs for which scalp maps indicated a left-right symmetric activity were fit using two dipoles constrained to be located symmetrically across the (corpus callosum) midline. The head model used for the analyses was the four-shell spherical 
model (BESA) that is coregistered with the average Montreal Neurological Institute (MNI) brain. Spherical dipole coordinates were converted to Talairach coordinates by DIPFIT using a nonlinear transform of MNI to Talairach implemented in the Matlab function "mni2tal.m" (http:// imaging.mrc-cbu.cam.ac.uk/imaging/MniTalairach). The brain structures where the dipoles were localized were identified using the Talairach atlas coordinates (Talairach \& Tournoux, 1988). The location solution was restricted to the gray matter, within a search range of $3 \mathrm{~mm}$.

\section{RESULTS}

\section{Behavioral Data}

The mean number of errors was 2.3 , which is $4.7 \%$ of the trials for the semantic instruction, and 2.2 (4.4\% of the trials) for the physical instruction. Given the risk of floor effects, no analyses of accuracies were made.

The analyses of RTs showed a main effect of letter case, $F(1,14)=5.91, p=.029$. When the letter case of the target was the same as that of the prime, RTs were shorter (i.e., $894 \mathrm{msec}$, on average) than when they were not (i.e., $919 \mathrm{msec}$ ). There was no interaction involving the factor letter case. RTs were shorter with the physical (789 $\mathrm{msec}$ ) than with the semantic instruction (906 msec), but this was just a trend, $F(1,14)=3.37, p=.088$, probably because instruction interacted with match (see below).

There was a main effect of match, $F(1,14)=12.42, p=$ .003 , indicating, as expected, that trials with a semantic mismatch were associated with longer RTs ( $867 \mathrm{msec}$ ) than match trials ( $829 \mathrm{msec})$. There was no interaction between the match effect and the semantic category. However, there was a significant interaction between match and instruction, $F(1,14)=19.91, p=.001$. Post hoc analyses for the semantic instruction revealed that the match effect of 80 msec observed between the match (866 msec) and the mismatch condition (946 $\mathrm{msec}$ ) was significant, $F(1$, $14)=19.51, p=.001$. In contrast, with the physical instruction, there was no significant difference between the match (790 msec) and the mismatch (788 msec) conditions.

A significant effect of target category was also found, $F(1$, $14)=4.57, p=.05$, as well as an interaction between target category and instruction, $F(1,14)=10.19, p=.007$. Post hoc analyses for the semantic instruction showed that animal targets were associated with shorter RTs (892 msec) than artifact targets (921 msec), $F(1,14)=10.28, p=$ .006. Post hoc analyses for the physical instruction showed no significant effect of target category.

Finally, to investigate the possibility that the match effect varies with the number of repetitions, we divided the trials into five levels of repetitions (0-20, 20-40, 40-60, 60-80, and 80-100) for the match and the mismatch conditions in the block of trials with the semantic instruction. This was made for the 7 subjects who went through this block first and for the 8 additional subjects who only had the semantic block. These 15 subjects had not viewed the words in the physical block before. Results of the analyses showed a main effect of match, $F(1,14)=37.38$, $p<.001$, which did not interact with repetition level, $F(4$, $56)=1.31, p=.29$ (see Figure 3B). However, there was a main effect of repetition level, $F(4,56)=21.10, p<.001$. Post hoc analyses showed that RTs decreased between 0 20 and 20-40 presentations (from 1016 to $878 \mathrm{msec}$, on average), $F(1,14)=18.26, p=.001$, between $20-40$ and 40-60 presentations (from 878 to $834 \mathrm{msec}$ ), $F(1,14)=$ $7.09, p=.02$, and between $40-60$ and $60-80$ presentations (from 834 to $811 \mathrm{msec}$ ), $F(1,14)=5.55, p=.03$, but they did not decrease significantly between 60-80 and 80-100 presentations (from 811 to $799 \mathrm{msec}$ ).

\section{Electrophysiological Data}

\section{General Description}

Figure 1 shows the target ERPs in the conditions in which they semantically matched the primes and in conditions where they did not. Negative is plotted upward in all figures. There was a clear match-mismatch difference with the semantic instruction (top) but not with the physical instruction (bottom). This difference appeared to be centered on an N400-like ERP similar to that found in Debruille and Renoult (2009). In the mismatch condition (in blue) at $\mathrm{Cz}$ electrode, this deflection peaked around $330 \mathrm{msec}$ postonset.

Figure 2 illustrates the target ERPs when their letter case was the same and when it was different from those of the primes. When the instruction was to assess letter case (bottom), greater positivities appeared for different than for same case that were centered on the P200 and the LPC. With the semantic instruction (top), this difference was temporally more extended, consisting essentially of a late slow wave that was also more positive for different than for same letter case.

P200. Statistical analyses of the mean voltage amplitudes of the ERPs in the P200 time window revealed a main effect of letter case for all subsets of electrodes, $F(1,14)=30.91$, $p<.001$ for the sagittal, $F(1,14)=22.25, p<.001$ for the parasagittal, and $F(1,14)=19.6, p=.001$ for the lateral subset, indicating that letter case that differed from those of the prime words were associated with greater P200 amplitudes than same letter case. This effect did not interact with instruction. An interaction with the electrode factor was found for all subsets, $F(3,42)=5.87, \varepsilon=.62, p=$ .009 for the sagittal, $F(6,78)=14.15, \varepsilon=.33, p<.001$ for the parasagittal, and $F(4,56)=23.5, \varepsilon=.37, p<$ .001 for the lateral subset. As indicated by Figure 2, the effect of letter case on the P200 was maximal at anterior and central sites but mostly absent at posterior sites. Post hoc analyses showed that the effect was significant at most sites $(F \geq 10.2, p \leq .007)$, that is, at $\mathrm{FP} 2 / 1, \mathrm{~F} 8 / 7, \mathrm{~F} 4 / 3, \mathrm{Fz}$, FT8/7, FC4/3, FCz, T4/3, C4/3, Cz, CP4/3, P3, and Pz. At the lateral subset, there was an interaction between letter 
Figure 1. Grand average ERPs to target words that semantically matched or mismatched the prime words. Negativity is up. With the semantic instruction (top), subjects had to respond according to whether the target word semantically matched the prime word. With the physical instruction (bottom), they had to respond according to whether the target word had the same letter case as the prime.

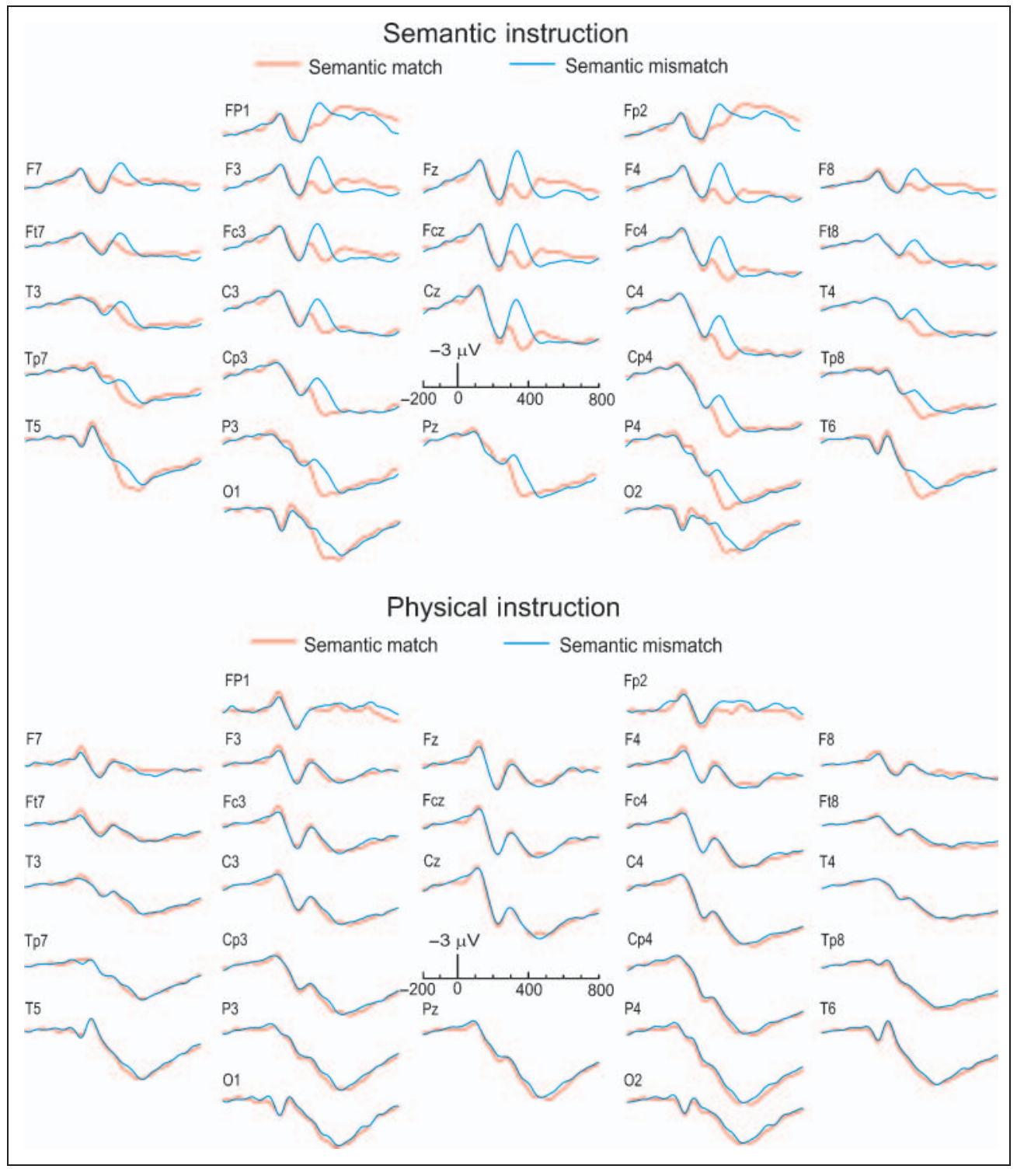

case, instruction, and the electrode factor, $F(4,56)=4.29$, $\varepsilon=.32, p=.044$. Post hoc analyses showed that, at F8 electrode, the effect of letter case was significantly greater with the physical than with the semantic instruction, $F(1$, $14)=6.07, p=.03$. Finally, there was no interaction between letter case and hemiscalp.

There was a main effect of instruction for all subsets of electrodes, $F(1,14)=16.13, p=.001$ for the sagittal, $F(1$, $14)=19.42, p=.001$ for the parasagittal, and $F(1,14)=$ $16.5, p=.001$ for the lateral subset, revealing that P200 amplitudes were significantly greater with the physical instruction. However, except for the aforementioned triple interaction with the electrode factor at the lateral subset, there was no significant interaction involving instruction and letter case.

We found a main effect of target category at the sagittal subset, $F(1,14)=7.74, p=.015$. This effect consisted of greater P200s for animal targets than artifact targets, especially at anterior sites, as illustrated by Figure 4, which shows the ERPs evoked by each category computed from all the trials of the experiment. This was supported by significant interactions between target category and electrode at the parasagittal, $F(6,78)=8.88, \varepsilon=.42, p<$ .001 , and at the lateral subset, $F(4,56)=31.85, \varepsilon=.39$, $p<.001$, and by a similar trend at the sagittal subset, $F(3$, $42)=3.39, p=.06$. At the parasagittal subset, there was an interaction between target category and hemiscalp, $F(1$, $14)=7.82, p=.015$, illustrating that the effect of category was greater over the right than the left hemiscalp. A similar difference at the lateral subset missed significance, $F(1,14)=$ $3.8, p=.07$. Post hoc analyses showed that animal targets produced greater P200s than artifact targets at many sites $(F \geq 4.6, p \leq .04)$, that is, at F8/7, F4/3, Fz, FT8, FC4/3, FCz, $\mathrm{Cz}, \mathrm{C} 4$, and T4. At FP2, this effect just missed significance, $F(1,14)=4.4, p=.06$. Interestingly, there was no interaction involving target category and instruction.

Finally, there was no effect of semantic match on the P200 nor any interaction involving this factor. 
N400-like potential. Statistical analyses of the mean voltage amplitudes of the N400-like potential revealed a main effect of match for all subsets of electrodes, $F(1,14)=$ $27.46, p<.001$ for the sagittal, $F(1,14)=24.97, p<$ .001 for the parasagittal, and $F(1,14)=17.04, p=.001$ for the lateral subset. The amplitudes of the N400-like potential were thus larger for semantic mismatches than matches. There was no significant interaction involving match and letter case. The match factor interacted with instruction at all subsets, $F(1,14)=12.31, p=.003$ for the sagittal, $F(1,14)=10.76, p=.005$ for the parasagittal, and $F(1,14)=8.73, p=.01$ for the lateral subset. Separate analyses for the semantic and the physical instruction showed that the match effect was significant with the semantic instruction, $F(1,14)=26.09, p<.001$ for the sagittal, $F(1,14)=22.59, p<.001$ for the parasagittal, and $F(1,14)=16.75, p=.001$ for the lateral subset, but not with the physical instruction (see Figure 1). With the semantic instruction, there were also interactions between the match and the electrode factor at the sagittal, $F(3,42)=$ $4.68 ; \varepsilon=.64, p=.019$, and the parasagittal subset, $F(6$, 78) $=6 ; \varepsilon=.33, p=.007$, and triple interactions between these factors and the hemiscalp factor at the parasagittal, $F(6,78)=3.04 ; \varepsilon=.51, p=.039$, and lateral subset, $F(4$, 56) $=3.96 ; \varepsilon=.59, p=.023$. These results revealed that the effect of semantic matching was greater at central sites and over the right than over the left hemiscalp. Figure $5 \mathrm{~A}$ shows the scalp voltage maps of the effect of semantic matching in the session with the semantic instruction that were obtained in the time window of the N400-like potential by subtracting match trials from mismatch trials. Post hoc analyses for each electrode site in the semantic instruction showed that the match effect was significant at all sites $(F \geq 7, p \leq .02)$.
Figure 2. Grand average ERPs to target words according to their letter case. For other details, see Figure 1.

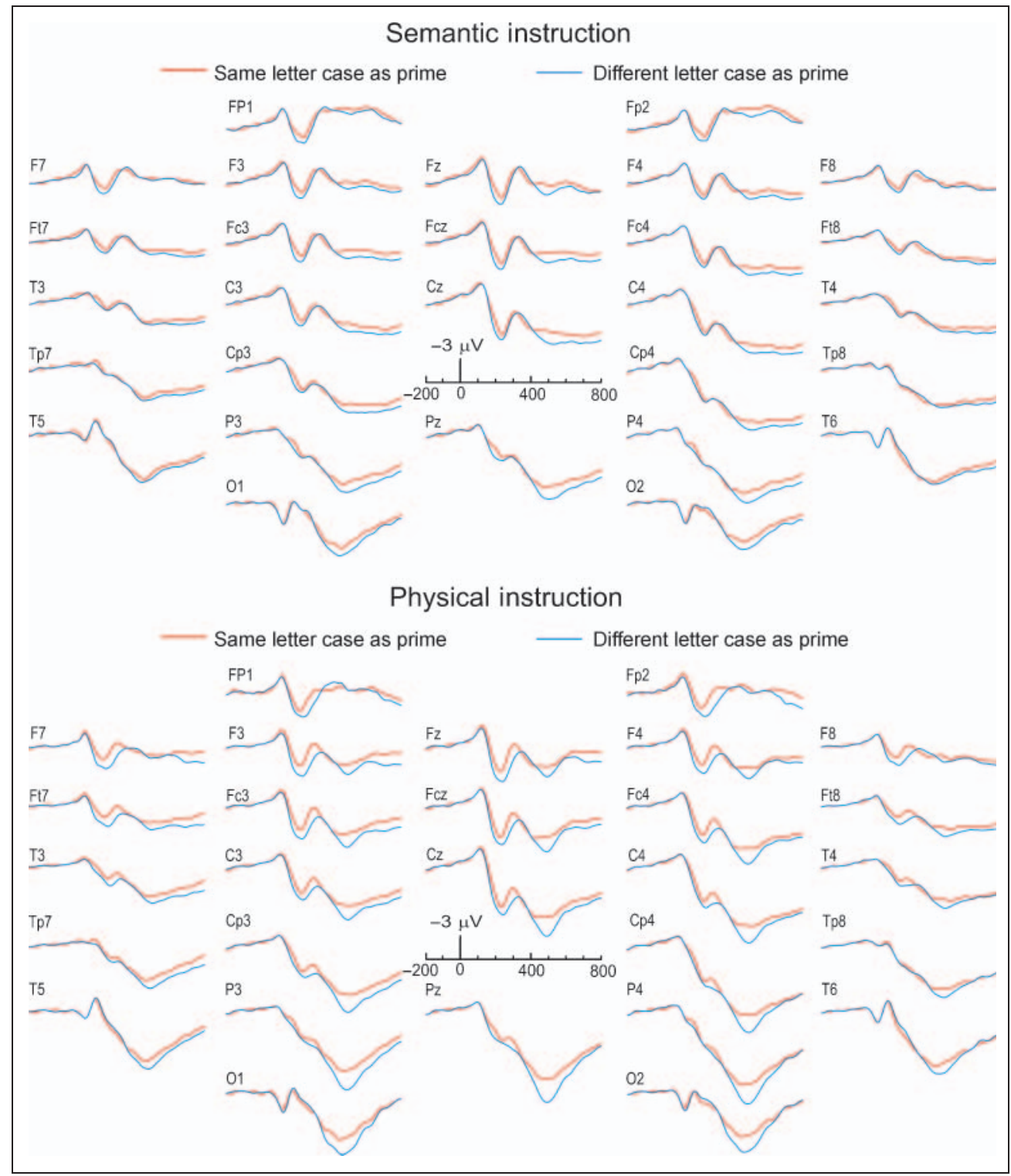




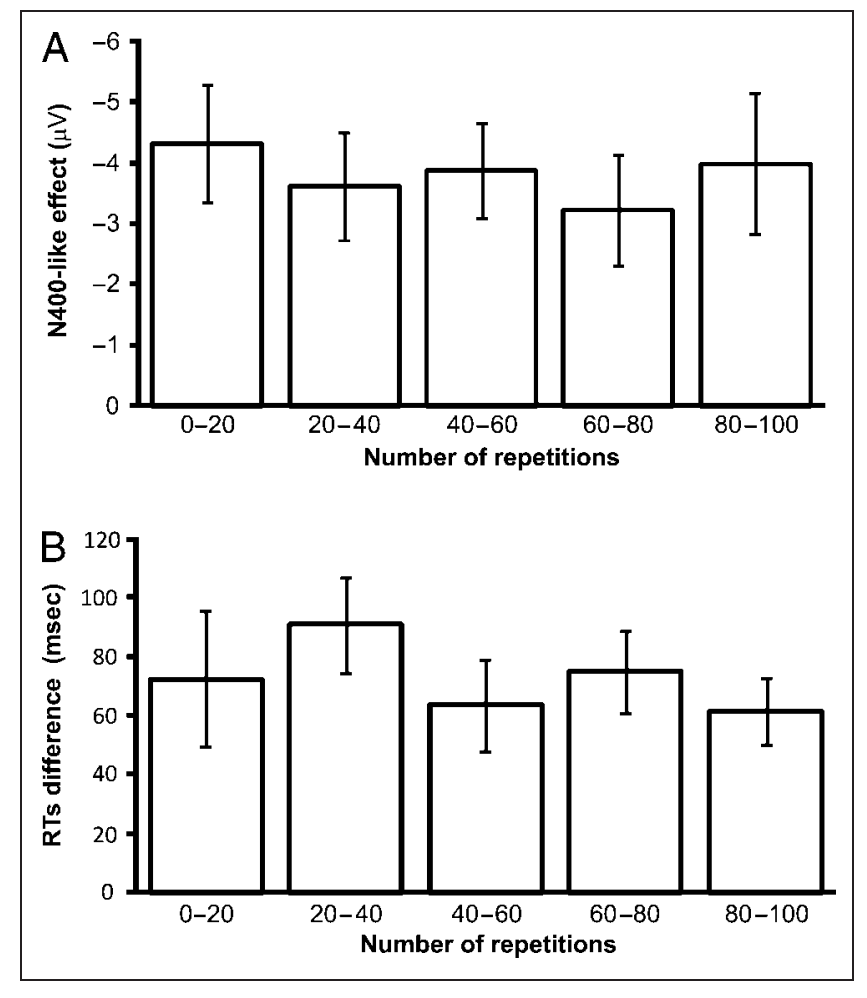

Figure 3. Mean size of the effect of semantic matching across repetition levels. Standard errors bars are shown for each level of repetition. (A) N400-like potential at electrode Cz. For each level of repetition, the mean voltage of grand average ERPs to semantically matching targets was subtracted from the mean to mismatching targets in the time window of the N400-like potential (265-405 msec after target onset). (B) RTs. For each level of repetition, the mean RT to semantically matching targets was subtracted from the mean RT to mismatching targets.

There was no significant interaction involving match and target category with either instruction, showing that the match effect was similar for both targets.

A main effect of target category was found at all subsets of electrodes, $F(1,14)=13.58, p=.002$ for the sagittal,
$F(1,14)=8.82, p=.01$ for the parasagittal, and $F(1,14)=$ $11.36, p=.005$ for the lateral subset. This effect did not interact with letter case. Contrary to our expectations, it did not interact with instruction either. Target category interacted with the electrode factor at all subsets, $F(3$, $42)=8.99, \varepsilon=.66, p=.001$ at the sagittal, $F(6,78)=$ 9.19, $\varepsilon=.33, p=.001$ at the parasagittal, and $F(4,56)=$ 28.07, $\varepsilon=.41, p<.001$ at the lateral subset, showing that animal targets were associated with a scalp distribution that differed from that associated to artifact targets. Post hoc analyses showed that artifact targets were significantly more negative than animal targets at most anterior and central sites $(F \geq 6, p \leq .03)$, that is, at FP2, F7/F8, F3/F4, Fz, FT7/FT8, FC3/FC4, at FCz, T3/T4, C3/C4, and at Cz and CP3 (see Figure 5B).

No main effect of letter case was found in the time window of the N400-like potential nor any interaction involving this factor.

Finally, to investigate the possibility that the match effect varies with the number of repetitions, we divided the trials into five levels of repetitions (0-20, 20-40, 40-60, 60-80, and 80-100) for the match and the mismatch conditions in the block of trials with the semantic instruction. This was made for the 7 subjects who went through this block first and for the 8 additional subjects who only had the semantic block. These 15 subjects had not viewed the words in the physical block before. Results of the analyses showed that the main effect of match was highly significant for all subsets of electrodes, $F(1,14)=33.08, p<.001$ for the sagittal, $F(1,14)=23.77, p<.001$ for the parasagittal, and $F(1,14)=16.05, p=.001$ for the lateral subset, but most importantly, there was not interaction involving match and repetition level, $F(4,56)=0.69, p=.57$ for the sagittal, $F(4,56)=2.15, p=.11$ for the parasagittal, and $F(4,56)=1.41, p=.26$ for the lateral subset (see Figure $3 \mathrm{~A}$ ). There was also no main effect of repetition level but interactions between this factor and electrodes for all subsets, $F(6,78)=3.07, \varepsilon=.37, p=.02$ for the sagittal,
Figure 4. Grand average ERPs to target words according to their semantic category (computed from all trials of the experiment). Animal targets (black lines) refer to the trials in which the word "chien" ("dog" in English) was used as a target. Artifact targets (gray lines) refer to the trials in which the word "table" (same spelling as in English) was used as a target.

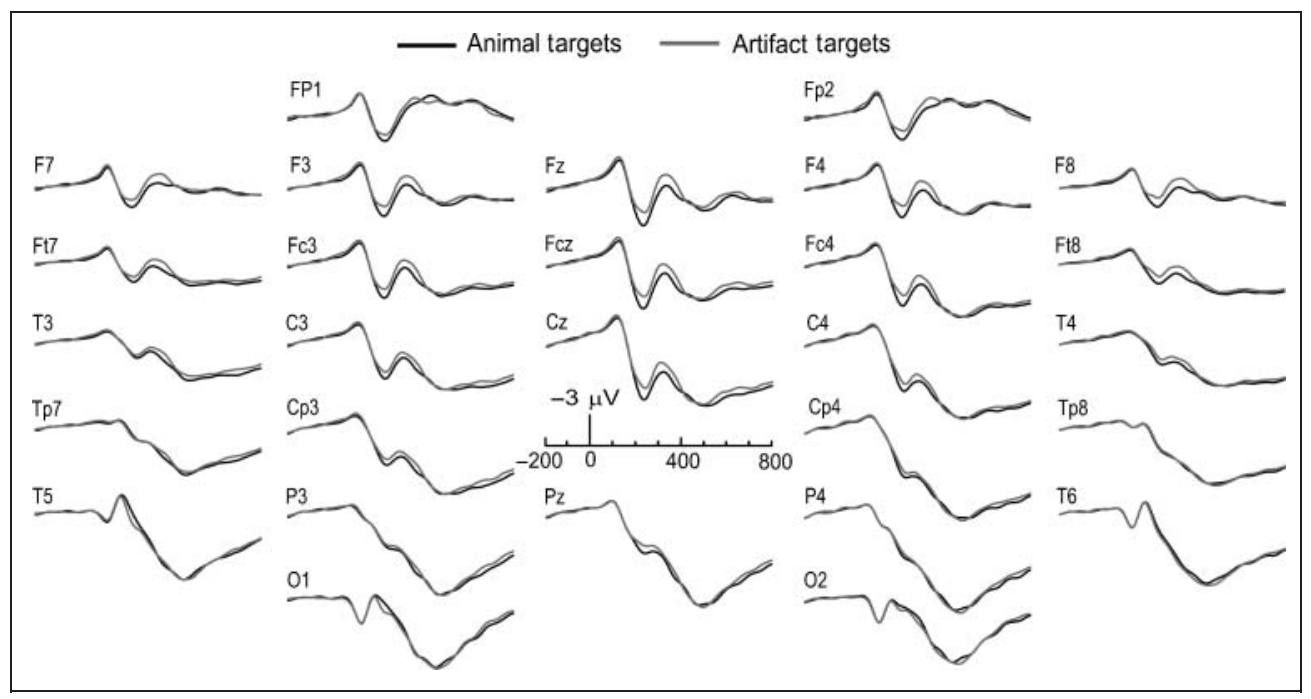


Figure 5. Spline interpolated isovoltage maps computed from subtractions of grand mean ERPs in the time window of the N400-like ERP. (A) Effect of semantic matching. The mean voltages for the matching trials were subtracted from the means for the mismatching trials in the semantic instruction block. (B) Effect of semantic category. The mean voltages obtained for all the animal targets were subtracted from the means obtained for all the artifact targets, using the data of the two instruction blocks.

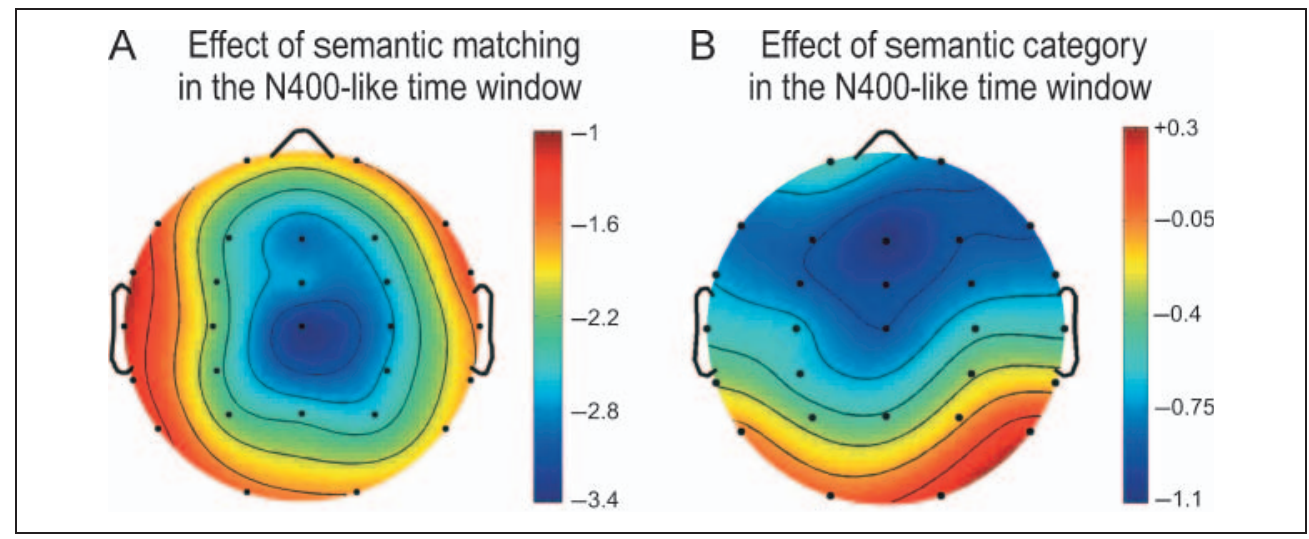

$F(6,78)=2.72, \varepsilon=.21, p=.02$ for the parasagittal, and $F(6,78)=4.89, \varepsilon=.29, p=.001$ for the lateral subset. Post hoc analyses showed that the effect of repetition level was significant at TP7, $F(4,56)=7.28, p=.002$, and tended to be so at $\mathrm{Pz}, F(4,56)=2.48, p=.08$, and $\mathrm{FP} 1, F(4,56)=2.32, p=.09$. Further analyses revealed that, at TP7, $F(1,14)=6.61, p=.02$, and Pz, $F(1$, $14)=5.27, p=.04$, mean voltage amplitudes were more positive after 20-40 than after 0-20 presentations.

Late Positive Complex. Statistical analyses of the mean voltage amplitudes of the ERPs in the LPC time window revealed a main effect of letter case for all subsets of electrodes, $F(1,14)=7.64, p=.015$ for the sagittal subset, $F(1$, $14)=10.29, p=.006$ for the parasagittal subset, and $F(1$, $14)=6.46, p=.023$ for the lateral subset, indicating that letter case that differed from those of the prime were associated with greater LPCs than same letter case (see Figure 2). Moreover, letter case interacted with the electrode factor at all subsets, $F(3,42)=11.6, \varepsilon=.61, p<.001$ for the sagittal, $F(6,78)=6.77, \varepsilon=.39, p=.002$ for the parasagittal, and $F(4,56)=4.19, \varepsilon=.44, p=.032$ for the lateral subset. Post hoc analyses showed that the effect was not significant at frontal sites but significant at all centro-posterior sites ( $F \geq 4.7, p \leq .04):$ FC4/FC3, FCz, T4/T3, C4/C3, Cz, TP8/TP7, CP4/CP3, T6/T5, P4/P3, Pz, and O2/O1.

There was also a main effect of task instruction, $F(1,14)=$ 9.96, $p=.007$ for the sagittal, $F(1,14)=14.76, p=.002$ for the parasagittal, and $F(1,14)=7.6, p=.015$ for the lateral subset. The physical instruction generated greater LPCs than the semantic instruction, as shown in Figure 2. There was however no significant interaction between instruction and letter case, and the interaction between these variables and the electrode factor was just a trend, $F(3,42)=2.5, p=$ .08 for the sagittal and $F(6,78)=2.4, p=.09$ for the parasagittal subset.

We found no main effect of semantic match in the LPC time window but a triple interaction between match, electrodes, and task instruction at the sagittal subset, $F(1,14)=$ $3.67, p=.048$. Post hoc analyses showed that the match effect was only significant with the semantic instruction at $\mathrm{Fz}, F(1,14)=6.84, p=.020$, and FCz, $F(1,14)=$ $4.99, p=.042$, electrodes. For these electrodes, mismatches were associated with greater LPC amplitudes than matches. However, one has to note that the semantic instruction block was characterized by variations in peak latency of the LPC. As indicated by Figure 1, the differences of amplitude between the match and the mismatch conditions at these two electrode sites may thus have been caused by shift in latency rather than real amplitude modulations.

Finally, no main effect of target category was found in the LPC time window nor any interaction involving this factor.

\section{Independent Component Analysis (ICA)}

The envtopoO function of EEGLAB (Onton et al., 2006; Delorme \& Makeig, 2004) was used to identify the five ICs that made the largest contribution to the effects of semantic matching (i.e., semantic mismatch - semantic match) and letter case (different letter case - same letter case as the prime) in each of the windows of measures of ERPs, which is that of the P200 (155-265 msec after target onset), N400-like potential (265-405 msec), and LPC (405-550 msec). For each of these ICs, we tested if their activity significantly differentiated each condition (i.e., semantic match vs. mismatch and same vs. different letter case). Source localization was also performed for each IC (see Methods section). Figure 6 represents the ICs that were significantly modulated by semantic matching (Figure 6A and C) and those that were significantly modulated by physical matching (Figure 6D).

P200. In the P200 time window, the activity of one IC was significantly greater in the semantic match condition than in the mismatch, $F(1,14)=8.7, p=.01$. This IC, IC7, had a right centro-parietal scalp distribution and accounted for $-62 \%$ of the match effect. Percent variance accounted for (PVAF) indicates by how many percent the scalp signal is reduced when a component is removed. 
This value can be negative when, like in the present case, subtracting the component actually increases signal variance. This is illustrated by Figure $6 \mathrm{C}$ which shows that during the P200 time window, this IC made a positive contribution to the match effect whereas the mean subtraction ERP was negative. Dipole source modeling revealed a source in the left posterior cingulate cortex, in the vicinity of Brodmann's areas (BA) 23 and 30 ( $x$ : $-2 ; y$ : -44 ; $z: 22)$. The residual variance of the dipole model (RVDM) was $1.5 \%$.

The activity of two other ICs was modulated by letter case, IC4, $F(1,14)=7.58, p=.02$, and IC8, $F(1,14)=$
$14.08, p=.002$. Their activity was greater when letter case of the target differed from that of the prime (see Figure 6D). IC4 projected maximally to central midline sites and contributed to $44 \%$ of the effect of letter case in the P200 time window, whereas IC8 had a right centro-parietal scalp distribution and a PVAF of $57 \%{ }^{2}$ Dipole source modeling for IC4 revealed that it was best modeled with a bilateral source in the inferior parietal cortex, in the vicinity of BA $40(x:-48 ; y:-41 ; z: 31)$, with an RVDM of $3 \%$ IC8 was located in the right fusiform gyrus, in the proximity of BA 20 and BA 36 ( $x: 46 ; y:-34 ; z:-17)$, with an RVDM of $4 \%$.

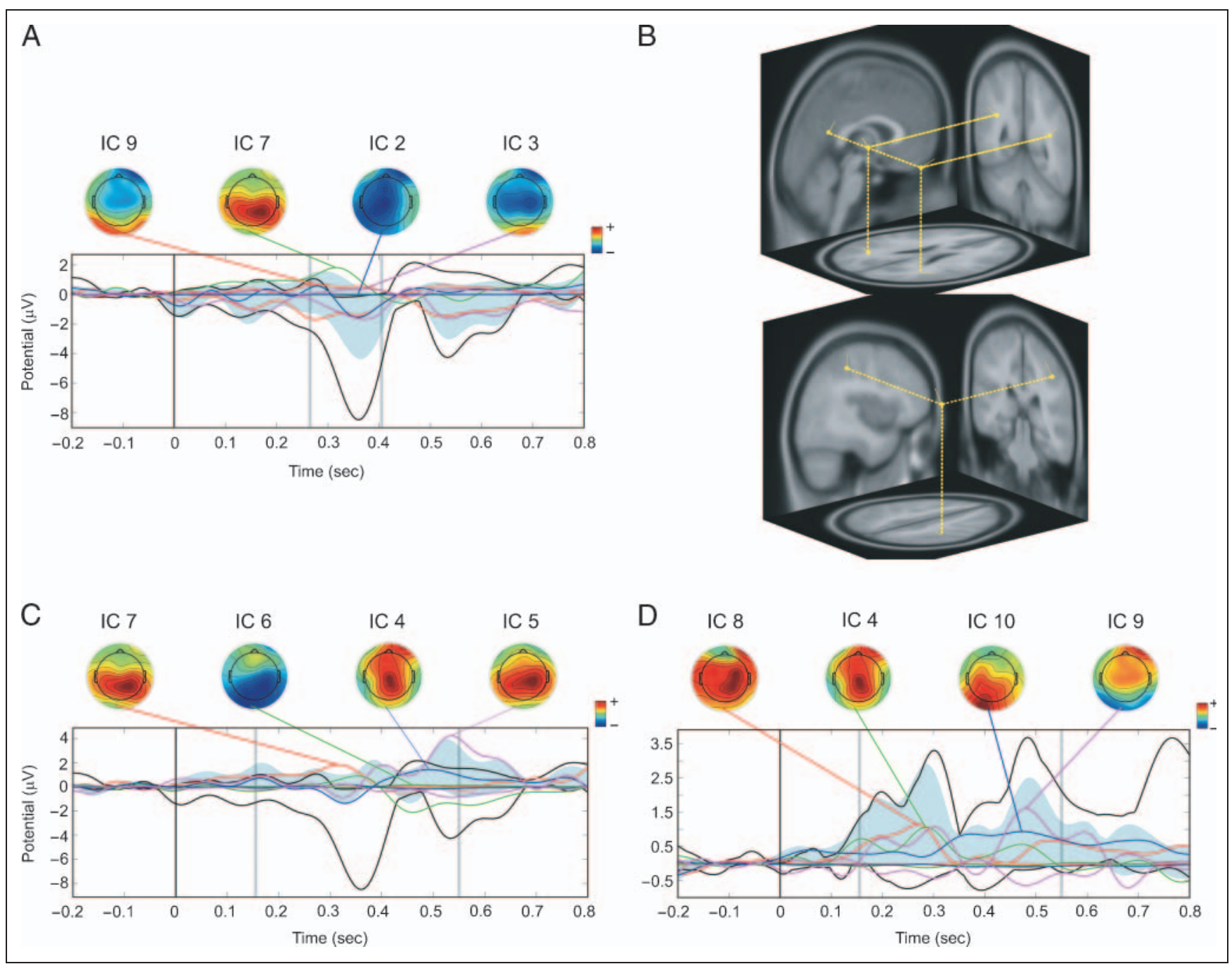

Figure 6. IC contributions to the effects of semantic matching (semantic mismatch - semantic match) and of physical matching (different letter case - same letter case as the prime). (A) ICs that were significantly modulated by semantic matching in the time window of the N400-like potential. The vertical black lines at time 0 indicate target words onset. The thick black lines show the envelope, that is, the most positive and negative values of the ERPs over all channels and at each time point. The blue traces show the envelopes of the contribution of the ICs represented. Each IC scalp map is connected to its data envelope by a color line that points to the moment of peak contribution to the ERP (see Delorme \& Makeig, 2004). (B) ECDs for IC3 (top) and IC2 (bottom). These two ICs were the greatest contributors to the match effect in the time window of the N400-like potential. IC3 accounted for $40 \%$ of the variance of the effect and was best fit by a bilateral generator in the superior temporal gyrus in the vicinity of BA $22(x:-36 ; y:-49 ; z: 23)$. IC2 accounted for $36 \%$ of the variance of the effect and was best fit by a generator in the right inferior parietal cortex in the vicinity of BA $40(x: 40 ; y:-30 ; z: 45)$. (C) ICs that were significantly modulated by semantic matching in the P200 and LPC time windows. (D) ICs that were significantly modulated by physical matching in the P200 and LPC time windows. 
N400-like potential. Four ICs were found to be significantly modulated by semantic matching in the time window of the N400-like potential (Figure 6A). IC2, $F(1,14)=$ $5.71, p=.03$, IC3, $F(1,14)=10.51, p=.006$, and IC9, $F(1$, 14) $=6.79, p=.02$, had a greater activity in the mismatch than in the match condition. In contrast, IC7, $F(1,14)=$ 9.6, $p=.008$, as described for the P200 time window, had a greater activity in the match than in the mismatch condition (PVAF: -36\%). IC2 had a left centro-parietal scalp distribution and a PVAF of $36 \%$. Its source was found in the right inferior parietal cortex, in the vicinity of BA 40 $(x: 40 ; y:-30 ; z: 45)$, with an RVDM of $8 \%$. IC3 had a bilateral parietal distribution centered on the vertex (with a slight right-sided asymmetry) and a PVAF of $40 \%$. Its source was identified as a bilateral generator in the superior temporal gyrus, in the vicinity of BA $22(x:-36 ; y:-49 ; z: 23)$, with an RVDM of $3.7 \%$ (see Figure 6B). IC9 had a bilateral frontal distribution and a PVAF of $23 \%$. Dipole source modeling revealed that this IC was best modeled by a midline dipole centered in the posterior cingulate gyrus, close to BA 30 and the anterior lobe of the cerebellum $(x: 0 ; y$ : -57 ; $z: 5$ ), with an RVDM of $6.8 \%$.

No ICs were found to be significantly modulated by letter case in the time window of the N400-like potential.

Late Positive Complex. The activity of three ICs was found to be significantly modulated by semantic matching in the LPC time window. IC6 had a greater activity in the mismatch than in the match condition, $F(1,14)=$ $7.89, p=.01$, whereas IC $4, F(1,14)=8.1, p=.01$, and IC $5, F(1,14)=5.5, p=.03$, were more active in the match than in the mismatch condition (see Figure 6C). IC6 had a right occipito-temporal scalp distribution and had a negative PVAF $(-24 \%)$, as this IC made a negative contribution to the match effect, whereas the mean subtraction ERP was positive during this period. This IC was located in the left cingulate gyrus, in the vicinity of BA $24(x:-3$; $y:-16 ; z: 35)$, with an RVDM of $1.7 \%$. In contrast to IC6, IC5 made a positive contribution to the match effect, whereas the mean subtraction ERP was negative (PVAF: $-30 \%)$. It had a right centro-parietal distribution and it was found to be located in the vicinity of the right hippocampus and parahippocampal gyrus $(x: 27 ; y:-45 ; z: 7)$, with an RVDM of $2.7 \%$. Finally, IC 4 had a central midline scalp distribution and a PVAF of $18 \%$. As indicated for the P200 time window, this IC was found to be generated in the inferior parietal cortex.

Two ICs were significantly modulated by letter case in the LPC time window (see Figure 6D). IC9, $F(1,14)=$ $6.12, p=.03$, and IC10, $F(1,14)=5.81, p=.03$, had a greater activity when letter case of the target differed from that of the prime. IC9, which had a frontal scalp distribution and was found to be generated in the posterior cingulate gyrus (BA 30), as described for the time window of the N400-like potential, had a PVAF of $25 \%$ in the LPC time window. IC 10 had a left occipito-temporal scalp distribution and a PVAF of $38 \%$. It was found to be generated in the posterior lobe of the left cerebellum ( $x$ : $-19 ; y$ : $-58 ; z$ : -18 ), with an RVDM of $6.8 \%$.

\section{DISCUSSION}

The present study aimed to test whether N400s and RTs could be used to study the semantic relations between two particular words, which, for this purpose, were massively repeated. We capitalized on the results obtained in a previous primed semantic categorization task (Debruille \& Renoult, 2009), in which RTs and N400-like potentials evoked by two highly repeated target words were found to be modulated by semantic matching and semantic category. Here, we tested if these effects could be observed when prime words were also highly repeated, so that each prime-target pair could be studied separately. Only two category words were used as primes and two exemplars as targets, resulting in a total of four possible pairs. To control for a potential contribution of physical matching, letter case of target words was manipulated. This allowed us to also verify that the N400-like effects on focus varied with task instruction. A block of trials with a physical instruction was contrasted to a block with a semantic instruction. As expected, target ERPs included an N400-like negativity. Significant effects of semantic matching and semantic category were found on RTs and ERPs. The effects of semantic matching were restricted to the semantic instruction. On the other hand, the P200 and the LPC, but not the N400-like ERP, were modulated by physical matching.

The fact that RTs were longer and the amplitude of the N400-like potential greater in the semantic mismatch than in the match condition confirms that participants did not simply retrieved the decision and the response associated to each of the four prime-target pairs but processed the words deeply. Moreover, the finding that the effects occurred only when subjects were instructed to focus on semantics illustrates the similarity between the present N400-like ERP and the classic N400. A suppression of N400 effects in case discrimination tasks has indeed been described by a number of studies (Chwilla et al., 1995; Deacon et al., 1991; Rugg, 1984). Furthermore, as commonly observed for the N400, the effect of semantic matching was significant throughout the scalp with a clear centro-parietal maximum (Curran, Tucker, Kutas, \& Posner, 1993). The use of exemplars as target words in the present study instead of categories as in Debruille and Renoult (2009) could explain this more classic distribution of the N400-like effect, whereas in our previous study, it was slightly more frontal. On the other hand, another difference with this study is that the effect of semantic matching on RTs and on N400-like potentials was similar for the two target words. This could be due to the fact that here these target words were typical exemplars of their category and were matched for their number of letters, frequency of usage, concreteness, imageability, subjective frequency, and syntactic category (both were nouns). This absence of interaction 
between match and category is important as it allows studying each of these effects with confidence that one was not contaminated by the other.

The possibility that the effect of semantic matching varies with the number of repetitions was eliminated by testing this effect through different levels of repetitions (0-20, 20-40, 40-60, 60-80, and 80-100) in the block of trials with the semantic instruction. Although the effect was highly significant, these analyses revealed that there was no interaction involving match and the level of repetition for both N400-like potentials and RTs. The match effect was thus comparable throughout the experiment. On the other hand, the analysis of RTs showed that while subjects were getting faster in word categorization throughout the experiment, this effect reached a floor between 60 and 80 presentations, when mean RT was about $800 \mathrm{msec}$. Therefore, even at this point, RTs were still much longer than the mean RTs obtained in simple choice RT tasks using words as stimuli (e.g., Kutas, McCarthy, \& Donchin, 1977). This is of critical importance as it further confirms that the words were still "deeply" processed after numerous repetitions. Finally, these analyses showed that the amplitude of the N400-like potential was not modulated by repetition level, except for two electrode sites where mean voltage was more positive after 20-40 than after 0-20 presentations. Studies having used multiple target words and lower levels of repetition have generally reported that N400 amplitude did not decrease further after two or three presentations (Van Strien et al., 2005; Kazmerski \& Friedman, 1997; Besson et al., 1992; Kounios \& Holcomb, 1992; Young \& Rugg, 1992). Our results can thus be taken as evidence that even after this early point where N400 is no longer modulated by additional repetitions, semantic effects can still be observed, at least in explicit semantic tasks.

On the other hand, the semantic category of target words significantly modulated RTs and ERPs. As previously found by a number of studies using multiple exemplars, RTs to artifact targets were longer than for animal targets with the semantic instruction (Debruille \& Renoult, 2009; Hoenig, Sim, Bochev, Herrnberger, \& Kiefer, 2008; Proverbio, Del Zotto, \& Zani, 2007; Paz-Caballero, Cuetos, \& Dobarro, 2006; Kiefer, 2001; Price \& Humphreys, 1989). Semantic category also modulated the P200 and the N400-like ERP in both instruction blocks. Incidentally, this allows emphasizing, as was done for the effect of semantic matching, that participants did not simply retrieved the decision associated with each pair of words. Had this been the case, no semantic category differences would have been present. It can thus be ascertained that target words were deeply processed. Animal targets produced greater P200s than artifact targets at fronto-central sites, especially over the right hemisphere. Similar early category effects on the P200 have been observed by a number of studies (Hoenig et al., 2008; Proverbio et al., 2007; Sitnikova, West, Kuperberg, \& Holcomb, 2006; Antal, Keri, Kovacs, Janka, \& Benedek, 2000), even if they were not always tested (Proverbio et al., 2007; Sitnikova et al., 2006) as most studies of category effects focus on late ERPs. Hoenig et al. (2008) proposed that such early effects would reflect the activation of conceptual features (e.g., visual or functional) rather than the integration or binding of features combinations. In the time window of the N400-like potential, ERPs to artifact targets were generally more negative than to animal targets over frontal and central sites. Similar frontal (Debruille \& Renoult, 2009; Proverbio et al., 2007; Antal et al., 2000) and central differences (Debruille \& Renoult, 2009; Proverbio et al., 2007; Kiefer, 2001, Experiment 1; Antal et al., 2000) between artifact and animal targets have been described by a number of N400 studies. In contrast, it has to be noted that in some other studies (Paz-Caballero et al., 2006; Kiefer, 2001, 2005), ERPs to names of artifacts were found more positive than those to names of animals at frontal sites. Although we did not find this reversed effect, the difference between artifact and animal targets was clearly attenuated at prefrontal sites, as in Debruille and Renoult (2009). Most importantly, similar effects of semantic category were found here with the semantic and the physical instruction. Paz-Caballero et al. (2006) also found similar category effects in a task where the processing of semantic category was explicit (i.e., semantic categorization) and in a task where it was not (i.e., gender decision). Nevertheless, we did not expect an effect of category with the physical instruction, in line with Kiefer (2005) and Sim and Kiefer (2005) where no or reduced effects of category were observed with repeated stimuli in tasks that did not require explicit semantic categorization. A posteriori, it seems that two important aspects of the present design could explain the presence of the category effect in our physical task. First, the constant presence of the two category words as primes may have prompted subjects to process target words as to their category even when this was not task relevant. Second, the use of typical exemplars throughout the task instead of multiple exemplars could lead to a greater resistance of the effect of category to repetition. Indeed, typical exemplars are thought to be more robustly represented in semantic memory and more efficiently recognized or retrieved than less typical exemplars (Patterson, 2007). Further studies should help quantifying the importance of these factors and gain a greater insight as to the modulation of the effect of category by repetition.

We did not find any effect of letter case on the N400like ERP, neither with the semantic nor with the physical instruction. This finding shows that this ERP, while being characterized by a peak latency earlier than that of the classic N400, is not influenced by physical matching or by a combination of semantic and physical matching processes. This strengthens the idea that it may be considered as a type of N400 potential. In contrast, the effect of physical matching modulated the P200 and the LPC. Although modulations of the LPC with physical matching have been repeatedly described (Moores et al., 2003; Besson \& Macar, 1986; Kutas \& Hillyard, 1980), similar modulations of the P200 have been more rarely studied, even if they can 
be observed in some ERP waveforms (e.g., in Moores et al., 2003; Kutas \& Hillyard, 1980). Using a letter identification task to study the time course of letter perception, Petit, Midgley, Holcomb, and Grainger (2006) recently showed that the P200 was sensitive to letter case, whereas a later component (i.e., the P260) was not.

The N400-like potential is thus not likely to be a visual N200. Letter case decision (Deacon et al., 1991) and also orthographic matching (Kramer \& Donchin, 1987) or form discrimination (Ritter, Simson, \& Vaughan, 1983) have been associated with increased N200 amplitudes in the mismatch conditions. The reason for which the letter case task was not associated with the modulation of an N200, as for instance in Deacon et al. (1991), may be the presence of the two prime words. As mentioned above, because these two words were category words, they might have maintained the structure of a semantic categorization task. Another possibility derives from the fact that P200 and N200 overlap. Thus, greater positivities for discrepant letter case may cancel greater N200s. However, the ICA did not reveal such opposite modulations for the effect of letter case in the P200 time window. Crucially, this analysis showed that no IC made a significant contribution to this effect in the time window of the N400-like potential.

The main generators of the effect of semantic matching in the time window of the N400-like ERP were similar to that commonly found for the N400 in nonrepeated conditions. The IC that made the greatest contribution to the effect of semantic matching had a scalp distribution centered on the vertex, similar to the main contributor of the effect found by Dien, Frishkoff, Cerbone, and Tucker (2003) with PCA or by Groppe (2007) with ICA. This IC was best fit with a bilateral source in the superior temporal gyrus (BA 22). Temporal lobe generators have been proposed to be the largest source of the N400 (Kutas et al., 2006; Van Petten \& Luka, 2006), with a greater contribution from the left than the right hemisphere. In addition to its anterior medial-temporal lobe generators described mostly by intracranial studies (Grunwald, Lehnertz, Heinze, Helmstaedter, \& Elger, 1998; Nobre \& McCarthy, 1995; Smith, Stapleton, \& Halgren, 1986), intracranial as well as fMRI, MEG, and high-density ERP studies have repeatedly described $\mathrm{N} 400$ generators in the vicinity of the auditory cortex, that is, in the superior temporal gyrus (Matsumoto, Iidaka, Haneda, Okada, \& Sadato, 2005; D’Arcy, Connolly, Service, Hawco, \& Houlihan, 2004; Halgren et al., 2002; Helenius et al., 2002; Helenius, Salmelin, Service, \& Connolly, 1998) or in the STS (Halgren et al., 1994, 2002; Elger et al., 1997; Simos, Basile, \& Papanicolaou, 1997; Guillem, N'Kaoua, Rougier, \& Claverie, 1995). When bilateral sources are reported, right hemisphere generators are sometimes active slightly after their left hemisphere homologs (Halgren et al., 2002; Helenius et al., 1998). More generally, fMRI studies that investigated the effect of semantic congruity using word pairs have shown that the superior and the middle temporal gyri were the most commonly active regions, along with inferior frontal regions (reviewed in Lau, Phillips, \& Poeppel, 2008; Van Petten \& Luka, 2006). The fact that no frontal generator was found for the match effect in the present study suggests that the cognitive processes associated with these regions, which include controlled retrieval and selection (Thompson-Schill, 2003; Buckner \& Wheeler, 2001), were not of critical importance in the present task. As only four word pairs were used throughout the experiment, it is likely that lexicosemantic representations of the words could be reactivated effortlessly.

The second greatest contributor to the effect of semantic matching in the time window of the N400-like potential projected most strongly to left centro-parietal sites and was localized in the right inferior parietal cortex (BA 40). Inferior parietal N400 generators have also been described by a number of studies (Guillem, Rougier, \& Claverie, 1999; Helenius et al., 1998; Guillem et al., 1995; Halgren et al., 1994; Nenov et al., 1991) and lesions of these areas including the TPJ are known to result in large N400 amplitude reduction (Friederici, Hahne, \& von Cramon, 1998; Swaab, Brown, \& Hagoort, 1997; Hagoort, Brown, \& Swaab, 1996). BA 40 , as observed in the present study, was found to be part of a network of common activations in working memory, semantic memory, and episodic-memory tasks (Nyberg, Forkstam, Petersson, Cabeza, \& Ingvar, 2002) and has been associated with active maintenance of verbal information (reviewed in Smith, Jonides, Marshuetz, \& Koeppe, 1998).

Another IC that was also modulated by semantic matching had a generator in the anterior medial-temporal lobe, in the vicinity of the hippocampus and parahippocampal gyrus, similar to that found by Debruille and Renoult (2009). However, unlike this study, it was found to make a greater contribution in the time window of the LPC than in the time window of the N400-like potential. More generally, differences between the present study and Debruille and Renoult (2009) in term of localization of the N400 match effect may be due to several differences in methodology, including the use of repeated primes and targets in the former case and the restriction of the match effect to animal targets in the latter case.

These analyses also showed that ICA can reveal modulations of ERPs with experimental variables that are nonvisible in mean voltage analyses because of source cancellations (e.g., Luck, 2005). This was the case in the P200 time window where one IC, which was found to be located in the left posterior cingulate gyrus, had a significantly greater activity in the match than in the mismatch condition. This modulation was barely visible and not significant in the analyses of mean voltages, likely because this effect was of opposite polarity as the mean ERPs during this period. Using simultaneous EEG-fMRI recordings, Eichele et al. (2005) found similar neural generators for P200 effects as in the present study, including the posterior cingulate gyrus and the supramarginal gyrus.

The N400-like ERP was characterized by its early peak latency as in Debruille and Renoult (2009; see also Simon et al., 2004; Karniski et al., 1993). Importantly, in this former study, this ERP was found to have the same onset latency as 
the N400 obtained in nonrepeated conditions. Furthermore, when comparing N400s evoked by the first 30 presentations of targets words to those evoked by the last 30 presentations, no latency difference was observed. The use of a high rate of repetition in simple categorization tasks therefore does not seem to allow the semantic processes indexed by $\mathrm{N} 400$ to occur earlier but simply to be active for a shorter duration. These results are concordant with observations showing that the onset latency of the N400 does not vary much in the visual modality, starting 200 to $250 \mathrm{msec}$ after word onset in various conditions (Hagoort, Hald, Bastiaansen, \& Petersson, 2004; van Berkum, Hagoort, \& Brown, 1999; Helenius et al., 1998). Helenius et al. (1998) proposed that a 250- to 350-msec time window, which corresponds to the first part of the N400, would be critical for the analysis of word meaning, whereas a later time window starting at about $350 \mathrm{msec}$ would be dedicated to postlexical contextual processes. Following this logic, the fact that the N400-like ERP was active for a shorter duration than in classical conditions and peaked $330 \mathrm{msec}$ after target onset may indicate that the use of massively repeated words rendered some of these later processes inactive. Alternatively, both of these processes may have been active for a shorter time.

In conclusion, the fact that effects of semantic matching and category were observed in the present study with only two prime and two target words suggests that semantic relations between individual words can be studied. The use of repetition could thus allow, at least in explicit semantic tasks, a dramatic reduction of the complexity of $\mathrm{N} 400$ protocols where only few carefully selected words could be included. The problems of category specificity and physical variance present when using large groups of words (Pulvermüller \& Shtyrov, 2006) could then be avoided. As previously mentioned, the study of associations between individual words could be relevant to examine between-subjects differences in associations. For instance, this type of protocols could be applied to the exploration of the deficits at integrating information that specifically disconfirm delusional beliefs in psychiatric patients. This could help to understand the persistence of such beliefs in front of contradictory evidence (Woodward, Moritz, Menon, \& Klinge, 2008; Debruille et al., 2007).

\section{Acknowledgments}

This study was supported by grant no. 194517-02 from the National Sciences and Engineering Research Council of Canada (NSERC) allocated to J. B. Debruille, who was supported by the scholarship 10084 from the Fonds de la Recherche en Santé du Québec (FRSQ). Louis Renoult was supported by the fellowship 13542 from the FRSQ. The authors thank Dr. Karsten Steinhauer and Dr. Debra Titone for helpful comments and suggestions on earlier drafts on this manuscript.

Reprint requests should be sent to J. Bruno Debruille, Department of Psychiatry, McGill University, Douglas Hospital Research Center, FBC-1, 6875 Boul LaSalle, Montreal, Quebec, Canada, H4H 1R3, or via e-mail: bruno.debruille@douglas.mcgill.ca.

\section{Notes}

1. We used the expression "semantic matching" as an equivalent to "semantic congruity" that better illustrates the process of matching prime and target words for their meaning in semantic categorization tasks.

2. Note that because ICs are not spatially orthogonal, the variance accounted for by all components together does not equal the sum of the variance accounted by each component alone (Groppe, Makeig, \& Kutas, 2008).

\section{REFERENCES}

Antal, A., Keri, S., Kovacs, G., Janka, Z., \& Benedek, G. (2000). Early and late components of visual categorization: An event-related potential study. Cognitive Brain Research, 9, 117-119.

Bell, A. J., \& Sejnowski, T. J. (1995). An informationmaximization approach to blind separation and blind deconvolution. Neural Computation, 7, 1129-1159.

Bentin, S., McCarthy, G., \& Wood, C. (1985). Event-related potentials, lexical decision, and semantic priming. Electroencephalography and Clinical Neurophysiology, 60, 353-355.

Besson, M., \& Kutas, M. (1993). The many facets of repetition: A cued-recall and event-related potential analysis of repeating words in same versus different sentence contexts. Journal of Experimental Psychology: Learning, Memory, and Cognition, 19, 1115-1133.

Besson, M., Kutas, M., \& Van Petten, C. (1992). An event-related potential analysis of semantic congruity and repetition effects in sentences. Journal of Cognitive Neuroscience, 4, 132-149.

Besson, M., \& Macar, F. (1986). Visual and auditory eventrelated potentials elicited by linguistic and nonlinguistic incongruities. Neuroscience Letters, 63, 109-114.

Blair, I. V., \& Banaji, M. R. (1996). Automatic and controlled processes in stereotype priming. Journal of Personality and Social Psychology, 6, 1142-1163.

Bonin, P., Méot, A., Aubert, L., Malardier, N., Niedenthal, P., \& Capelle-Toczek, M. C. (2003). Normes de concrétude, de valeur d'imagerie, de fréquence subjective et de valence émotionnelle pour 866 mots. L'Année Psychologique, 104, 683-721.

Buckner, R. L., \& Wheeler, M. E. (2001). The cognitive neuroscience of remembering. Nature Reviews Neuroscience, 2, 624-634.

Chwilla, D. J., Brown, C. M., \& Hagoort, P. (1995). The N400 as a function of the level of processing. Psychophysiology, 32, 274-285.

Content, A., Mousty, P., \& Radeau, M. (1990). Une base de données lexicales informatisée pour le français écrit et parlé. L'Année Psychologique, 90, 551-566.

Curran, T., Tucker, D. M., Kutas, M., \& Posner, M. I. (1993). Topography of the N400: Brain electrical activity reflecting semantic expectancy. Electroencephalography and Clinical Neurophysiology, 88, 188-209.

D’Arcy, R. C., Connolly, J. F., Service, E., Hawco, C. S., \& Houlihan, M. E. (2004). Separating phonological and semantic processing in auditory sentence processing: A high-resolution event-related brain potential study. Human Brain Mapping, 22, 40-51.

Deacon, D., Breton, F., Ritter, W., \& Vaughan, H. G., Jr. (1991). The relationship between N2 and N400: Scalp distribution, stimulus probability, and task relevance. Psychophysiology, 28, 185-200. 
Debruille, J. B., Kumar, N., Saheb, D., Chintoh, A., Gharghi, D., Lionnet, C., et al. (2007). Delusions and processing of discrepant information: An event-related brain potential study. Schizophrenia Research, 89, 261-277.

Debruille, J. B., \& Renoult, L. (2009). Effects of semantic matching and of semantic category on reaction time and N400 that resist numerous repetitions. Neuropsychologia, 47, 506-517.

Dehaene, S., Jobert, A., Naccache, L., Ciuciu, P., Poline, J. B., Le Bihan, D., et al. (2004). Letter binding and invariant recognition of masked words: Behavioral and neuroimaging evidence. Psychological Science, 15, 307-313.

Dehaene, S., Naccache, L., Cohen, L., Le Bihan, D., Mangin, J.-F., Poline, J.-B., et al. (2001). Cerebral mechanisms of word masking and unconscious repetition priming. Nature Neuroscience, 4, 752-758.

Delorme, A., \& Makeig, S. (2004). EEGLAB: An open source toolbox for analysis of single-trial EEG dynamics including independent component analysis. Journal of Neuroscience Methods, 134, 9-21.

Devlin, J. T., Moore, C. J., Mummery, C. J., Gorno-Tempini, M. L., Phillips, J. A., Noppeney, U., et al. (2002). Anatomic constraints on cognitive theories of category specificity. Neuroimage, 15, 675-685.

Dien, J., Frishkoff, G. A., Cerbone, A., \& Tucker, D. M. (2003). Parametric analysis of event-related potentials in semantic comprehension: Evidence for parallel brain mechanisms. Cognitive Brain Research, 15, 137-153.

Dien, J., Khoe, W., \& Mangun, G. R. (2007). Evaluation of PCA and ICA of simulated ERPs: Promax vs. Infomax rotations. Human Brain Mapping, 28, 742-763.

Eichele, T., Specht, K., Moosmann, M., Jongsma, M. L., Quiroga, R. Q., Nordby, H., et al. (2005). Assessing the spatiotemporal evolution of neuronal activation with single-trial event-related potentials and functional MRI. Proceedings of the National Academy of Sciences, U.S.A., 102, 17798-17803.

Electrode Nomenclature Committee. (1991). Modified expanded 10-20 system. American EEG society. Journal of Clinical Neurophysiology, 35, 200-202.

Elger, C. E., Grunwald, T., Lehnertz, K., Kutas, M., Helmstaedter, C., Brockhaus, A., et al. (1997). Human temporal lobe potentials in verbal learning and memory processes. Neuropsychologia, 35, 657-667.

Finnigan, S., Humphreys, M. S., Dennis, S., \& Geffen, G. (2002). ERP "old/new" effects: Memory strength and decisional factor(s). Neuropsychologia, 40, 2288-2304.

Folstein, J. R., \& Van Petten, C. (2008). Influence of cognitive control and mismatch on the N2 component of the ERP: A review. Psychophysiology, 45, 152-170.

Friederici, A. D., Hahne, A., \& von Cramon, D. Y. (1998). Firstpass versus second-pass parsing processes in a Wernicke's and a Broca's aphasic: Electrophysiological evidence for a double dissociation. Brain and Language, 62, 311-341.

Greenhouse, G. W., \& Geisser, S. (1959). On methods of analysis of profile data. Psychometrika, 24, 1582-1589.

Groppe, D. M. (2007). Common independent components of the P3b, N400, and P600 ERP components to deviant linguistic events. Unpublished $\mathrm{PhD}$ dissertation, University of California, San Diego.

Groppe, D. M., Makeig, S., \& Kutas, M. (2008). Independent component analysis of event-related potentials. Cognitive Science, 6.1, 1-44.

Grunwald, T., Lehnertz, K., Heinze, H. J., Helmstaedter, C., \& Elger, C. E. (1998). Verbal novelty detection within the human hippocampus proper. Proceedings of the National Academy of Sciences, U.S.A., 95, 3193-3197.

Guillem, F., N'Kaoua, B., Rougier, A., \& Claverie, B. (1995). Intracranial topography of event-related potentials (N400/P600) elicited during a continuous recognition memory task. Psychophysiology, 32, 382-392.

Guillem, F., Rougier, A., \& Claverie, B. (1999). Short- and long-delay intracranial ERP repetition effects dissociate memory systems in the human brain. Journal of Cognitive Neuroscience, 11, 437-458.

Hagoort, P., Brown, C. M., \& Swaab, T. Y. (1996). Lexical-semantic event-related potential effects in patients with left hemisphere lesions and aphasia and patients with right hemisphere lesions without aphasia. Brain, 119, 627-649.

Hagoort, P., Hald, L., Bastiaansen, M., \& Petersson, K. M. (2004). Integration of word meaning and world knowledge in language comprehension. Science, 304, 438-441.

Halgren, E., Baudena, P., Heit, G., Clarke, J. M., Marinkovic, K., \& Clarke, M. (1994). Spatio-temporal stages in face and word processing. I. Depth-recorded potentials in the human occipital, temporal and parietal lobes. Journal of Physiology (Paris), 88, 1-50.

Halgren, E., Dhond, R. P., Christensen, N., Van Petten, C., Marinkovic, K., Lewine, J. D., et al. (2002). N400-like magnetoencephalography responses modulated by semantic context, word frequency, and lexical class in sentences. Neuroimage, 17, 1101-1116.

Helenius, P., Salmelin, R., Service, E., \& Connolly, J. F. (1998). Distinct time courses of word and context comprehension in the left temporal cortex. Brain, 121, 1133-1142.

Helenius, P., Salmelin, R., Service, E., Connolly, J. F., Leinonen, S., \& Lyytinen, H. (2002). Cortical activation during spoken-word segmentation in nonreading-impaired and dyslexic adults. Journal of Neuroscience, 22, 2936-2944.

Hoenig, K., Sim, E. J., Bochev, V., Herrnberger, B., \& Kiefer, M. (2008). Conceptual flexibility in the human brain: Dynamic recruitment of semantic maps from visual, motor, and motionrelated areas. Journal of Cognitive Neuroscience, 10, 1799-1814.

Holcomb, P. J. (1988). Automatic and attentional processing: An event-related brain potential analysis of semantic priming. Brain and Language, 35, 66-85.

Holcomb, P. J., Kounios, J., Anderson, J. E., \& West, W. C. (1999). Dual coding, context availability, and concreteness effects in sentence comprehension: An electrophysiological investigation. Journal of Experimental Psychology: Learning, Memory, and Cognition, 25, 721-742.

Holcomb, P. J., Reder, L., Misra, M., \& Grainger, J. (2005). The effects of prime visibility on ERP measures of masked priming. Cognitive Brain Research, 24, 155-172.

Jung, T. P., Makeig, S., Westerfield, M., Townsend, J., Courchesne, E., \& Sejnowski, T. J. (2001). Analysis and visualization of single-trial event-related potentials. Human Brain Mapping, 14, 166-185.

Karniski, W., Vanderploeg, R., \& Lease, L. (1993). "Virtual N400" and slow wave topography to auditory sentence incongruence. Brain and Language, 44, 58-79.

Kazmerski, V. A., \& Friedman, D. (1997). Effect of multiple presentations of words on event-related potential and reaction time repetition effects in Alzheimer's patients and young and older controls. Neuropsychiatry, Neuropsychology, and Behavioral Neurology, 10, 32-47.

Kiefer, M. (2001). Perceptual and semantic sources of categoryspecific effects: Event-related potentials during picture and word categorization. Memory \& Cognition, 29, 100-116.

Kiefer, M. (2005). Repetition-priming modulates categoryrelated effects on event-related potentials: Further evidence for multiple cortical semantic systems. Journal of Cognitive Neuroscience, 17, 199-211.

Kounios, J., \& Holcomb, P. J. (1992). Structure and process in semantic memory: Evidence from event-related brain potentials and reaction times. Journal of Experimental Psychology: General, 121, 459-479. 
Kounios, J., \& Holcomb, P. J. (1994). Concreteness effects in semantic processing: ERP evidence supporting dual-coding theory. Journal of Experimental Psychology: Learning, Memory, and Cognition, 20, 804-823.

Kramer, A. F., \& Donchin, E. (1987). Brain potentials as indices of orthographic and phonological interaction during word matching. Journal of Experimental Psychology: Learning, Memory, and Cognition, 13, 76-86.

Kutas, M., \& Hillyard, S. A. (1980). Reading senseless sentences: Brain potentials reflect semantic incongruity. Science, 207, 203-205.

Kutas, M., \& Hillyard, S. A. (1984). Brain potentials during reading reflect word expectancy and semantic association. Nature, 307, 161-163.

Kutas, M., McCarthy, G., \& Donchin, E. (1977). Augmenting mental chronometry. Science, 197, 792-795.

Kutas, M., Van Petten, C., \& Kluender, R. (2006).

Psycholinguistics electrified II: 1994-2005. In M. Traxler \& M. A. Gernsbacher (Eds.), Handbook of psycholinguistics (2nd ed., pp. 659-724). New York: Elsevier.

Lau, E. F., Phillips, C., \& Poeppel, D. (2008). A cortical network for semantics: (De)constructing the N400. Nature Reviews Neuroscience, 9, 920-933.

Luck, S. J. (2005). Ten simple rules for designing ERP experiments. In T. C. Handy (Ed.), Event-related potentials A methods handbook (pp. 17-32). Cambridge, MA: MIT Press.

Lust, S. A., \& Bartholow, B. D. (2009). Self-reported and P3 event-related potential evaluations of condoms: Does what we say match how we feel? Psychophysiology, 46, 420-424.

Makeig, S., Jung, T. P., Bell, A. J., Ghahremani, D., \& Sejnowski, T. J. (1997). Blind separation of auditory event-related brain responses into independent components. Proceedings of the National Academy of Sciences, U.S.A., 94, 10979-10984.

Makeig, S., Westerfield, M., Jung, T. P., Covington, J., Townsend, J., Sejnowski, T. J., et al. (1999). Functionally independent components of the late positive event-related potential during visual spatial attention. The Journal of Neuroscience, 19, 2665-2680.

Martin, A. (2001). Functional neuroimaging of semantic memory. In R. Cabeza \& A. Kingstone (Eds.), Handbook of functional neuroimaging of cognition (pp. 153-186). Cambridge: MIT Press.

Matsumoto, A., Iidaka, T., Haneda, K., Okada, T., \& Sadato, N. (2005). Linking semantic priming effect in functional MRI and event-related potentials. Neuroimage, 24, 624-634.

Mitchell, P., Andrews, S., \& Ward, P. B. (1993). Event-related potential indices of semantic and repetition priming: Effects of changing sentence context. Psychophysiology, 30, 496-509.

Mitchell, P. I., Andrews, S., Fox, A. M., Catts, S. V., Ward, P. B., \& McConaghy, N. (1991). Active and passive attention in schizophrenia: An ERP study of information processing in a linguistic task. Biological Psychology, 32, 101-124.

Moores, K. A., Clark, C. R., Hadfield, J. L., Brown, G. C., Taylor, D. J., Fitzgibbon, S. P., et al. (2003). Investigating the generators of the scalp recorded visuo-verbal P300 using cortically constrained source localization. Human Brain Mapping, 18, 53-77.

Nenov, V. I., Halgren, E., Smith, M. E., Badier, J. M., Ropchan, J., Blahd, W. H., et al. (1991). Localized brain metabolic response correlated with potentials evoked by words. Behavioral Brain Research, 44, 101-104.

Nobre, A. C., \& McCarthy, G. (1995). Language-related field potentials in the anterior-medial-temporal lobe: II. Effects of word type and semantic priming. Journal of Neuroscience, 15, 1090-1098.

Nyberg, L., Forkstam, C., Petersson, K. M., Cabeza, R., \& Ingvar, M. (2002). Brain imaging of human memory systems: Between-systems similarities and within-system differences. Cognitive Brain Research, 13, 281-292.
Onton, J., Westerfield, M., Townsend, J., \& Makeig, S. (2006). Imaging human EEG dynamics using independent component analysis. Neuroscience and Biobehavioral Reviews, 30, 808-822.

Patterson, K. (2007). The reign of typicality in semantic memory. Philosophical Transactions of the Royal Society of London, Series B, Biological Sciences, 362, 813-821.

Paz-Caballero, D., Cuetos, F., \& Dobarro, A. (2006). Electrophysiological evidence for a natural/artifactual dissociation. Brain Research, 1067, 189-200.

Petit, J. P., Midgley, K. J., Holcomb, P. J., \& Grainger, J. (2006). On the time course of letter perception: A masked priming ERP investigation. Psychonomic Bulletin \& Review, 13, 674-681.

Picton, T. W., Bentin, S., Berg, P., Donchin, E., Hillyard, S. A., Johnson, R., Jr., et al. (2000). Guidelines for using human event-related potentials to study cognition: Recording standards and publication criteria. Psychophysiology, 37, 127-152.

Price, C. J., \& Humphreys, G. W. (1989). The effects of surface detail on object categorization and naming. Quarterly Journal of Experimental Psychology, 41, 797-827.

Proverbio, A. M., Del Zotto, M., \& Zani, A. (2007). The emergence of semantic categorization in early visual processing: ERP indices of animal vs. artifact recognition. BMC Neuroscience, 8, 24.

Pulvermüller, F., \& Shtyrov, Y. (2006). Language outside the focus of attention: The mismatch negativity as a tool for studying higher cognitive processes. Progress in Neurobiology, 79, 49-71.

Ritter, W., Simson, R., \& Vaughan, H. G., Jr. (1983). Eventrelated potential correlates of two stages of information processing in physical and semantic discrimination tasks. Psychophysiology, 20, 168-179.

Robin, F. (2006). French norms of imagery for pictures, for concrete and abstract words. Canadian Journal of Experimental Psychology, 60, 221-236.

Rugg, M. D. (1984). Event-related potentials in phonological matching tasks. Brain and Language, 23, 225-240.

Rugg, M. D. (1985). The effects of semantic priming and word repetition on event-related potentials. Psychophysiology, 22, 642-647.

Rugg, M. D. (1995). ERP studies of memory. In M. D. Rugg \& M. G. H. Coles (Eds.), Electrophysiology of mind, eventrelated brain potentials and cognition (pp. 132-170). Oxford, UK: Oxford University Press.

Rugg, M. D., Brovedani, P., \& Doyle, M. C. (1992). Modulation of event-related potentials (ERPs) by word repetition in a task with inconsistent mapping between repetition and response. Electroencephalography and Clinical Neurophysiology, 84, 521-531.

Scherg, M. (1990). Fundamentals of dipole source potential analysis. In F. Grandon, M. Hoke, \& G. L. Romani (Eds.), Auditory evoked magnetic fields and potentials (Vol. 6, pp. 40-69). Basel: Karger.

Sim, E. J., \& Kiefer, M. (2005). Category-related brain activity to natural categories is associated with the retrieval of visual features: Evidence from repetition effects during visual and functional judgments. Cognitive Brain Research, 24, 260-273.

Simon, G., Bernard, C., Largy, P., Lalonde, R., \& Rebai, M. (2004). Chronometry of visual word recognition during passive and lexical decision tasks: An ERP investigation. International Journal of Neuroscience, 114, 1401-1432.

Simos, P. G., Basile, L. F. H., \& Papanicolaou, A. C. (1997). Source localization of the N400 response in a sentencereading paradigm using evoked magnetic Welds and magnetic resonance imaging. Brain Research, 762, 29-39. 
Sitnikova, T., West, W. C., Kuperberg, G. R., \& Holcomb, P. J. (2006). The neural organization of semantic memory: Electrophysiological activity suggests feature-based segregation. Biological Psychology, 71, 326-340.

Smith, E. E., Jonides, J., Marshuetz, C., \& Koeppe, R. A. (1998). Components of verbal working memory: Evidence from neuroimaging. Proceedings of the National Academy of Sciences, U.S.A., 95, 876-882.

Smith, M. E., Stapleton, J. M., \& Halgren, E. (1986). Human medial temporal lobe potentials evoked in memory and language tasks. Electroencephalography and Clinical Neurophysiology, 63, 145-159.

Swaab, T., Brown, C., \& Hagoort, P. (1997). Spoken sentence comprehension in aphasia: Event-related potential evidence for a lexical integration deficit. Journal of Cognitive Neuroscience, 9, 39-66.

Talairach, J., \& Tournoux, P. (1988). Co-planar stereotaxic atlas of the buman brain. Stuttgart: Thieme.

Thompson-Schill, S. L. (2003). Neuroimaging studies of semantic memory: Inferring "how" from "where." Neuropsychologia, 41, 280-292.

Tulving, E. (1972). Episodic and semantic memory. In E. Tulving \& W. Donaldson (Eds.), Organization of memory (pp. 381-403). New York: Academic Press.

van Berkum, J. J., Hagoort, P., \& Brown, C. M. (1999). Semantic integration in sentences and discourse: Evidence from the N400. Journal of Cognitive Neuroscience, 11, 657-671.

Van Petten, C., Kutas, M., Kluender, R., Mitchiner, M., \& McIsaac, H. (1991). Fractionating the word repetition effect with event-related potentials. Journal of Cognitive Neuroscience, 3, 131-150.

Van Petten, C., \& Luka, B. J. (2006). Neural localization of semantic context effects in electromagnetic and hemodynamic studies. Brain and Language, 97, 279-293.

Van Strien, J. W., Hagenbeek, R. E., Stam, C. J., Rombouts, S. A., \& Barkhof, F. (2005). Changes in brain electrical activity during extended continuous word recognition. Neuroimage, 26, 952-959.

West, W. C., \& Holcomb, P. J. (2000). Imaginal, semantic, and surface-level processing of concrete and abstract words: An electrophysiological investigation. Journal of Cognitive Neuroscience, 12, 1024-1037.

Woodward, S. H., Ford, J. M., \& Hammett, S. C. (1993). N4 to spoken sentences in young and older subjects. Electroencephalography and Clinical Neurophysiology, 87, 306-320.

Woodward, T. S., Moritz, S., Menon, M., \& Klinge, R. (2008). Belief inflexibility in schizophrenia. Cognitive Neuropsychiatry, 13, 267-277.

Young, M. P., \& Rugg, M. D. (1992). Word frequency and multiple repetition as determinants of the modulation of event-related potentials in a semantic classification task. Psychophysiology, 29, 664-676. 